International Journal of Bifurcation and Chaos, Vol. 12, No. 6 (2002) 1403-1409

(c) World Scientific Publishing Company

\title{
STABILIZATION OF GLOBAL INVARIANT SETS FOR CHAOTIC SYSTEMS: AN ENERGY BASED CONTROL APPROACH
}

\author{
LAURENT LAVAL and NACER K. M'SIRDI \\ Laboratoire de Robotique de Paris, UPMC - UVSQ - CNRS URA 17778, \\ 10-12, av. de l'Europe, 78140 Vélizy, France
}

Received May 15, 2001; Revised June 14, 2001

\begin{abstract}
This paper presents a new control approach for steering trajectories of three-dimensional nonlinear chaotic systems towards stable stationary states or time-periodic orbits. The proposed method mainly consists in a sliding mode-based control design that is extended by an explicit consideration of system energy as basis for both controller design and system stabilization. The control objective is then to regulate the energy with respect to a shaped nominal representation implicitly related to system trajectories. In this paper, we establish some theoretical results to introduce the control design approach referred to as Energy based Sliding Mode Control (ESMC for short). Then, some capabilities of the proposed approach are illustrated through examples related to the chaotic circuit of Chua.
\end{abstract}

Keywords: Control of chaos; nonlinear systems; sliding mode control; invariant sets.

\section{Introduction}

Motivated by potential applications in physics, electrical engineering, communication theory and many others fields, the control of chaotic systems has received an increasing interest. In this way, many ideas and methods for controlling chaos with an external forcing input have been proposed in the past: OGY method, adaptive control, time-delay feedback control, predictive Poincaré control, etc. (see [Ditto et al., 1995; Chen \& Dong, 1998; Chen, 1999] and references therein). Control of chaotic systems using sliding mode control theory was addressed through several papers (e.g. [Yu, 1996; Lenz \& Berstecher, 1997]). In particular, in [Yau et al., 2000] was proposed a sliding mode control scheme to asymptotically track target orbits for a class of uncertain chaotic systems. As a result, for any given initial conditions, the states of the controlled system converge to and lie within a neighborhood of the switching function related to tracking, robustness and performance specifications. However, these conventional sliding mode control schemes need an explicit definition of target trajectories as general solutions of a nominal system representation. Moreover, neither criteria nor constraints for selecting target trajectories are explicitly formulated. Therefore, in this paper we address the problem of controlling chaos using a sliding mode based control, in such a way that trajectory selection burden is avoided by means of constraints expressed as energetic criteria. More precisely, we propose an approach which aims at designing Variable Structure Controllers for three-dimensional nonlinear (chaotic) systems, to stabilize global invariant sets included in a selected two-dimensional subspace of the state space. Then, according to the controlled system asymptotic properties and PoincaréBendixon theorem, the (controlled) system trajectories may be driven to an equilibrium point, an asymptotically stable limit cycle or be itself a (stable) periodic orbit. For this purpose, the proposed approach consists in a sliding mode based 
control design that we extend by an explicit consideration of system energy as basis for both controller design and system stabilization. The control objective is then to regulate the energy with respect to a shaped nominal representation which is implicitly related to system trajectories. As a result, the Variable Structure Control scheme, referred to as Energy based Sliding Mode Control (ESMC for short), leads to an automatic generation of the (controlled) system trajectories and a good robustness image to modelling uncertainties.

This paper is organized as follows. Section 2 deals with the theoretical framework of the control approach. Section 3 illustrates some capabilities of the proposed Energy based Sliding Mode Control approach through examples related to the chaotic circuit of Chua. Finally, in Sec. 4, some concluding remarks are given.

\section{Theoretical Framework}

This paper considers the class of nonlinear autonomous systems of the form,

$$
\dot{X}=F(X, U)=f(X)+U
$$

where $X \in \mathbb{R}^{3}$ is the state vector partitionned as $X=\left[\begin{array}{ll}X_{1} & X_{2}^{T}\end{array}\right]^{T}$ with $^{1} X_{1} \in \Sigma_{1}\left(X_{1}\right) \subset \mathbb{R}, X_{2} \in$ $\Sigma_{2}\left(X_{2}\right) \subset \mathbb{R}^{2} . X(0) \in \mathbb{R}^{3}$ is the vector of initial conditions, $f(X)=\left[f_{1}\left(X_{1}, X_{2}\right) \quad f_{2}^{T}\left(X_{1}, X_{2}\right)\right]^{T}$ with $f_{1}\left(X_{1}, X_{2}\right) \in C^{1}(\mathbb{R})$ and $f_{2}\left(X_{1}, X_{2}\right) \in C^{1}(\mathbb{R} \times \mathbb{R})$, $U \in \mathbb{R}^{3}$ is the vector of control inputs.

From sliding mode control theory (e.g. [Utkin, 1977; DeCarlo et al., 1996; Slotine \& Li, 1991]), it is well known that by designing or choosing a switching surface $S(X)=0$ so that the system restricted to the surface has a desired global behavior (such as stability, tracking, regulation, etc....), and by applying a switched control law ${ }^{2}$ of the form:

$$
u=\hat{f}(X)+K \operatorname{sign}(S)
$$

(where $\hat{f}(X)$ represents the equivalent system dynamics on the sliding surface) then, for any initial conditions, the controlled system trajectories converge to in finite time and are maintained in an invariant set $\Omega_{0}=\{X / S(X)=0\}$.

In what follows, we propose some new results related to stabilization of an extended invariant set
$\Omega_{I S}$, included in the same subspace as $X_{2}$ and not restricted to $\{0\}$. For this purpose, we consider the following assumptions,

Assumption A1. The system is, at least, locally observable and controllable.

Assumption A2. The energy of the system can be represented by a Lyapunov function $V$ which can be split into two positive parts $V_{1}$ and $V_{2}(V=$ $\left.V_{1}+V_{2}\right)$ related to scalar positive functions $V_{T}\left(X_{1}\right)$ and $V_{I S}\left(X_{2}\right)$ respectively (i.e. $V_{1}=g_{1}\left(V_{T}\left(X_{1}\right)\right)$ and $\left.V_{2}=g_{2}\left(V_{I S}\left(X_{2}\right)\right)\right)$.

Assumption A3. Scalar positive functions $V_{T}\left(X_{1}\right)$ and $V_{I S}\left(X_{2}\right)$ have continuous first derivatives which can be expressed as,

$$
\begin{gathered}
\dot{V}_{T}=\Psi_{1} \dot{X}_{1} X_{1} \\
\dot{V}_{I S}=\dot{X}_{2}^{T} \Psi_{2} X_{2}
\end{gathered}
$$

where $\Psi_{1} \in \mathbb{R}^{+*}$ (i.e. strictly positive) and $\Psi_{2}$ is a $2 \times 2$ diagonal matrix with strictly positive real values along the diagonal.

\section{Now we can state our main results.}

Lemma 1. Consider the autonomous system (1) and Assumptions A1-A3. Moreover, consider the following control structure,

$$
U=\left[\begin{array}{ll}
u_{T} & u_{I S}^{T}
\end{array}\right]^{T}
$$

with,

$$
\begin{aligned}
u_{T} & =-\Gamma_{1} \operatorname{sign}\left(V_{T}\right) X_{1}-\hat{f}_{1}\left(X_{1}, X_{2}\right) \\
u_{I S} & =-\Gamma_{2} \operatorname{sign}\left(V_{I S}-V_{I S}^{*}\right) X_{2}-\hat{f}_{2}\left(0, X_{2}\right)
\end{aligned}
$$

where $u_{T} \in \mathbb{R}, u_{I S} \in \mathbb{R}^{2 \times 1}$. $\hat{f}_{1}\left(X_{1}, X_{2}\right)$ and $\hat{f}_{2}(0$, $\left.X_{2}\right)$ are respectively a continuous function and a vector of continuous functions which represent equivalent system dynamics. $\Gamma_{1}$ is a strictly positive real value and $\Gamma_{2}$ is a $2 \times 2$ diagonal matrix with strictly positive real values (to be tuned). $V_{I S}^{*}$ is a positive constant which characterizes a desired magnitude of energy. Then,

(i) all solutions of the controlled system asymptotically converge to and are maintained in a global invariant set $\Omega_{I S}$ included in the same subspace as $X_{2}$ and defined $b y^{3}\left\langle\overline{V_{I S}-V_{I S}^{*}}\right\rangle=$ 0 ,

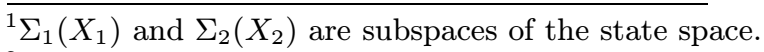

${ }^{2}$ So that away from the surface $S(X)=0$, the tangent vectors of the state trajectories point towards the surface.

${ }^{3}\langle\bar{\bullet}\rangle$ : averaged value of $\bullet$.
} 
(ii) $V_{T}$ converges to 0 in a finite time,

(iii) the energy of the controlled system converges to a neighborhood $\varepsilon_{I S}$ of $V_{I S}^{*}$.

Proof. With respect to Assumption A2, let us consider a Lyapunov function candidate $V=V_{1}+V_{2}$ with $V_{1}=(1 / 2) V_{T}^{2}$ and $V_{2}=(1 / 2)\left(V_{I S}-V_{I S}^{*}\right)^{2}$.

First, let us focus on $V_{1}=(1 / 2) V_{T}^{2}$. Then,

$$
\dot{V}_{1}=\dot{V}_{T} V_{T} .
$$

From Assumption A3 and system definition (1), $\dot{V}_{T}$ can be expressed as,

$$
\begin{aligned}
\dot{V}_{T} & =\Psi_{1} \dot{X}_{1} X_{1} \\
\Leftrightarrow & \dot{V}_{T}=\Psi_{1}\left(f_{1}\left(X_{1}, X_{2}\right)+u_{T}\right) X_{1} .
\end{aligned}
$$

Substituting (5) into (8) leads to,

$$
\begin{aligned}
\dot{V}_{T}= & \Psi_{1}\left(f_{1}\left(X_{1}, X_{2}\right)-\Gamma_{1} \operatorname{sign}\left(V_{T}\right) X_{1}\right. \\
& \left.-\hat{f}_{1}\left(X_{1}, X_{2}\right)\right) X_{1} \\
\Leftrightarrow \dot{V}_{T}= & \Psi_{1}\left(f_{1}\left(X_{1}, X_{2}\right)-\hat{f}_{1}\left(X_{1}, X_{2}\right)\right) X_{1} \\
& -\Psi_{1} \Gamma_{1} \operatorname{sign}\left(V_{T}\right) X_{1}^{2}
\end{aligned}
$$

as $\hat{f}_{1}\left(X_{1}, X_{2}\right)$ represents equivalent system dynamics according to $f_{1}\left(X_{1}, X_{2}\right)$, then, the averaged value $\left\langle\overline{f_{1}\left(X_{1}, X_{2}\right)-\hat{f}_{1}\left(X_{1}, X_{2}\right)}\right\rangle$ is zero. Thus, in the mean,

$$
\dot{V}_{T} \simeq-\Psi_{1} \Gamma_{1} \operatorname{sign}\left(V_{T}\right) X_{1}^{2} .
$$

Therefore,

$$
\dot{V}_{1}=\dot{V}_{T} V_{T} \simeq-\Psi_{1} \Gamma_{1} \operatorname{sign}\left(V_{T}\right) X_{1}^{2} V_{T} \leq 0 .
$$

Then, $\dot{V}_{1}$ is negative semi-definite and $V_{1}$ is positive definite. Consequently, from sliding mode theory [Utkin, 1977; DeCarlo et al., 1996], $V_{T}$ converges to a vicinity of 0 in finite time $t_{1}>0$. Moreover, $X_{1}$ is bounded and also goes to a vicinity of zero. Then,

Now, let us focus on $V_{2}=(1 / 2)\left(V_{I S}-V_{I S}^{*}\right)^{2}$.

$$
\dot{V}_{2}=\dot{V}_{I S}\left(V_{I S}-V_{I S}^{*}\right) .
$$

From Assumption A3 and system definition (1), $\dot{V}_{I S}$ can be expressed as,

$$
\begin{aligned}
\dot{V}_{I S} & =\dot{X}_{2}^{T} \Psi_{2} X_{2} \\
\Leftrightarrow & \dot{V}_{I S}=\left(f_{2}\left(X_{1}, X_{2}\right)+u_{I S}\right)^{T} \Psi_{2} X_{2} .
\end{aligned}
$$

Substituting (6) into (14) leads to,

$$
\begin{aligned}
\dot{V}_{I S}= & \left(f_{2}\left(X_{1}, X_{2}\right)-\Gamma_{2} \operatorname{sign}\left(V_{I S}-V_{I S}^{*}\right) X_{2}\right. \\
& \left.-\hat{f}_{2}\left(0, X_{2}\right)\right)^{T} \Psi_{2} X_{2} \\
\Leftrightarrow \dot{V}_{I S}= & \left(f_{2}\left(X_{1}, X_{2}\right)-\hat{f}_{2}\left(0, X_{2}\right)\right)^{T} \Psi_{2} X_{2} \\
& -\left(\Gamma_{2} \operatorname{sign}\left(V_{I S}-V_{I S}^{*}\right) X_{2}\right)^{T} \Psi_{2} X_{2} .
\end{aligned}
$$

Recalling that, $\hat{f}_{2}\left(0, X_{2}\right)$ represents equivalent system dynamics ${ }^{4}$ when $X_{1}$ is zero and that $V_{T}$ goes to a vicinity of 0 in a finite time $t_{1}$, then,

$$
\left\langle\overline{f_{2}\left(X_{1}, X_{2}\right)-\hat{f}_{2}\left(0, X_{2}\right)}\right\rangle=0 \quad \forall t \geq t_{1} .
$$

Thus, in the mean,

$$
\begin{aligned}
\dot{V}_{I S} & \cong-\left(\Gamma_{2} \operatorname{sign}\left(V_{I S}-V_{I S}^{*}\right) X_{2}\right)^{T} \Psi_{2} X_{2} \quad\left(\forall t \geq t_{1}\right) \\
\dot{V}_{2} & =\dot{V}_{I S}\left(V_{I S}-V_{I S}^{*}\right) \\
& \simeq-\left(\Gamma_{2} \operatorname{sign}\left(V_{I S}-V_{I S}^{*}\right) X_{2}\right)^{T} \Psi_{2} X_{2}\left(V_{I S}-V_{I S}^{*}\right)
\end{aligned}
$$

Thus, for $t \geq t_{1}, \dot{V}_{2}$ is negative semi-definite and $V_{2}$ is positive definite. Consequently, the sliding surface defined by $S_{2}(X)=V_{I S}-V_{I S}^{*}=0$ is attractive and $V_{I S}$ converges to a vicinity of $V_{I S}^{*}$ in a finite time $t_{2} \geq t_{1}>0$.

Therefore, we can conclude that, in a finite time $t_{2} \geq t_{1}>0$, the controlled system trajectories are driven to and maintained in an invariant set $\Omega_{I S}$ defined by $\left\langle\overline{V_{I S}-V_{I S}^{*}}\right\rangle=0$ and included in $\Sigma_{2}\left(X_{2}\right)$.

Moreover, as the system energy is bounded and its representation (related to $V_{T}\left(X_{1}\right)$ and $V_{I S}\left(X_{2}\right)$ ) goes to a vicinity of $V_{I S}^{*}$ in a finite time, this energy also converges to a neighborhood $\varepsilon_{I S}$ of $V_{I S}^{*}$ (related to discrepancy between the actual energy and its representation).

Remark. In (6), $u_{I S}$ is not explicitly related to $V_{T}$. This means that $u_{I S}$ does not contribute directly to the convergence of $V_{T}\left(X_{1}\right)$ and $X_{1}$ to 0 . This leads us to propose the following result.

Corollary 1. Lemma 1 holds when replacing $V_{I S}$ in (6) by a positive scalar function $V$ of the form $V=V_{T}+V_{I S}$

Proof. In proof of Lemma 1, let us replace the positive function $V_{2}=(1 / 2)\left(V_{I S}-V_{I S}^{*}\right)^{2}$ by,

$$
V_{2}=\frac{1}{2}\left(V-V_{I S}^{*}\right)^{2} \quad \text { with } V=V_{T}+V_{I S} .
$$

${ }^{4}$ According to $f_{2}\left(X_{1}, X_{2}\right)$. 
Then,

$$
\begin{aligned}
\dot{V}_{2} & =\dot{V}\left(V-V_{I S}^{*}\right) \\
\Leftrightarrow \dot{V}_{2} & =\left(\dot{V}_{T}+\dot{V}_{I S}\right)\left(V_{T}+V_{I S}-V_{I S}^{*}\right) .
\end{aligned}
$$

Due to $u_{T}, V_{T}$ and $\dot{V}_{T}$ converge to a vicinity of 0 in a finite time $t_{1}>0$. Therefore, in the mean,

$$
\dot{V}_{2} \cong\left(\dot{V}_{I S}\right)\left(V_{I S}-V_{I S}^{*}\right) \quad \forall t \geq t_{1} .
$$

End of proof is then straightforward.

\section{Example: Control of Chua's Circuit Using ESMC Approach}

This section aims at illustrating some capabilities of ESMC approach to steer third-order chaotic system trajectories to selected stationary points or stable limit cycles. For this purpose, we consider the chaotic circuit of Chua (e.g. [Madan, 1993; Yang \& Chua, 1998]) depicted in Fig. 1.

This system can be represented as follows (see [Yang \& Chua, 1998]),

$$
\left\{\begin{array}{l}
\dot{v}_{1}=\frac{1}{C_{1}}\left[G\left(v_{2}-v_{1}\right)-f_{d}\left(v_{1}\right)\right] \\
\dot{v}_{2}=\frac{1}{C_{2}}\left[G\left(v_{1}-v_{2}\right)+i_{3}\right] \\
\dot{i}_{3}=\frac{1}{L}\left[-v_{2}-R_{0} i_{3}\right]
\end{array}\right.
$$

where $G=1 / R$ and $f_{d}\left(v_{1}\right)$ is a piecewise continuous function related to Chua's diode characteristic. This function can be expressed as,

$$
f_{d}\left(v_{1}\right)=G_{b} v_{1}+\frac{1}{2}\left(G_{a}-G_{b}\right)\left[\left|v_{1}+E\right|-\left|v_{1}-E\right|\right]
$$

where $E$ represents the breakpoint voltage of Chua's diode.

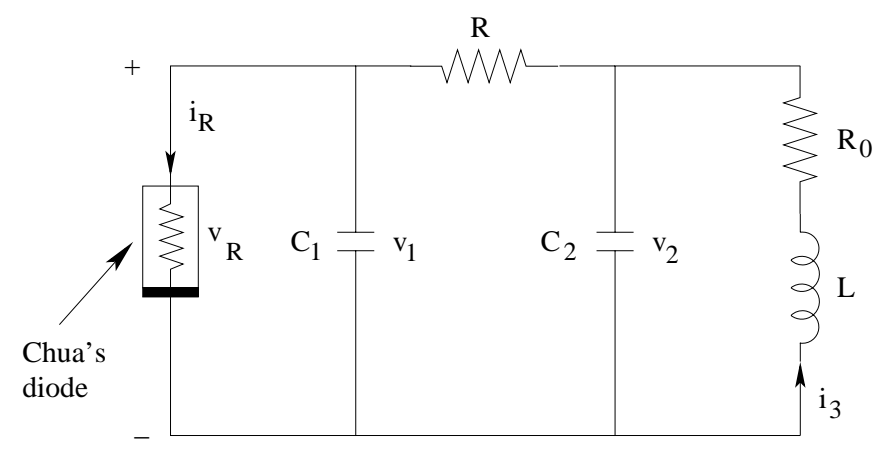

Fig. 1. Chua's circuit.
Without loss of generality, we assume $R_{0}=0$. Then, using appropriated transformations, the Chua's circuit can be formulated as a dimensionless system of the form [Yang \& Chua, 1998],

$$
\dot{X}=f(X) \Leftrightarrow\left\{\begin{array}{l}
\dot{x}=\alpha\left(y-x-f_{d}(x)\right) \\
\dot{y}=x-y+z \\
\dot{z}=-\beta y
\end{array}\right.
$$

where $X=[x, y, z]^{T}$ is the state vector with $x=$ $v_{1} / E, y=v_{2} / E, z=i_{3} / E G, \alpha=C_{2} / C_{1}, \beta=$ $C_{2} R^{2} / L$ and $f_{d}(x)=b x+(1 / 2)(a-b)(|x+1||x-1|)$ with $a=R G_{a}$ and $b=R G_{b}$.

Finally, by considering the circuit of Fig. 1, the energy of system (22) can be expressed as,

$$
E_{S}=\frac{1}{2} C_{1} v_{1}^{2}+\frac{1}{2} C_{2} v_{2}^{2}+\frac{1}{2} L i_{3}^{2}
$$

or, using dimensionless notations,

$$
E=\frac{1}{2} x^{2}+\frac{1}{2} \alpha y^{2}+\frac{1}{2} \frac{\alpha}{\beta} z^{2} .
$$

Application of ESMC approach to the Chua's circuit is considered as follows.

Preliminary Remark. According to ESMC approach, state vector $X \in \mathbb{R}^{3}$ must be partitioned into two $X_{1} \in \mathbb{R}$ and $X_{2} \in \mathbb{R}^{2}$. For this purpose, let us remark that this partition is of crucial importance as it completely defines the subspace of the state space in which the global invariant set has to be stabilized. Moreover, from Assumption A2, split of Lyapunov function $V$ (representing the system energy) is directly related to state vector partitioning. Therefore, this partitioning requires to take into account the physical properties of the system as some subspaces of the state space may not admit any stable invariant set with finite energy.

By considering the previous remark, possible transfers of energy between Chua's circuit components lead us, for instance, to deal with $X_{1}=x$ and $X_{2}=\left[\begin{array}{ll}y & z\end{array}\right]^{T}$. Then, according to (26) and Assumptions A2 and A3, let us consider the following Lyapunov function $V(X)$ and its split form components $V_{1}\left(X_{1}\right)$ and $V_{2}\left(X_{2}\right)$ to represent the energy of the system:

$$
V(X)=V_{1}\left(X_{1}\right)+V_{2}\left(X_{2}\right)
$$


where,

$$
\begin{aligned}
& V_{1}\left(X_{1}\right)= \frac{1}{2} V_{T}^{2} \quad \text { with } V_{T}=\frac{1}{2} x^{2} \\
& V_{2}\left(X_{2}\right)= \frac{1}{2}\left(V_{I S}-V_{I S}^{*}\right)^{2} \\
& \text { with } V_{I S}=\frac{1}{2} \alpha y^{2}+\frac{1}{2} \frac{\alpha}{\beta} z^{2} \\
& V_{I S}^{*} \text { is a constant. }
\end{aligned}
$$

Then, from Lemma 1, the controlled chaotic system of Chua is given by

$$
\left\{\begin{array}{l}
\dot{x}=\alpha\left(y-x-f_{d}(x)\right)+u_{T} \\
\dot{y}=x-y+z+u_{I S_{1}} \\
\dot{z}=-\beta y+u_{I S_{2}}
\end{array}\right.
$$

with,

$$
\begin{aligned}
u_{T} & =-\Gamma_{1} \operatorname{sign}\left(V_{T}\right) x-\hat{f}_{1}(x, y, z) \\
u_{I S_{1}} & =-\Gamma_{11} \operatorname{sign}\left(V_{I S}-V_{I S}^{*}\right) y-\hat{f}_{21}(0, y) \\
u_{I S_{2}} & =-\Gamma_{22} \operatorname{sign}\left(V_{I S}-V_{I S}^{*}\right) z-\hat{f}_{22}(0, z) .
\end{aligned}
$$

Assume the equivalent system dynamics can be defined as,

$$
\begin{aligned}
\hat{f}_{1}(x, y, z) & =\hat{\alpha}\left(y-x-\widehat{f_{d}(x)}\right) \\
\hat{f}_{21}(0, y) & =z-y \\
\hat{f}_{22}(0, y) & =\hat{\beta} y .
\end{aligned}
$$

Then, by considering system parameters: $\alpha=$ $10, \beta=14.87, a=-1.27$ and $b=-0.68$, initial conditions $x(0)=0.2, y(0)=0.1$ and $z(0)=-0.01$, control law gains $\Gamma_{1}=20, \Gamma_{2}=I_{2}, \hat{\alpha}=\alpha, \hat{\beta}=\beta$, we obtain simulation results related to the following two cases.

First case. Let us consider a targeted magnitude of system energy $V_{I S}^{*}=0$. Obviously, a such null value implies that the targeted (global) invariant set is restricted to zero value. ${ }^{5}$ As shown in Figs. 2 and 3 , the system trajectory first goes, in a finite time $t_{1} \cong 0.4 s$, to $(y, z)$ plane (see Fig. 3 ) before converging, in a finite time $t_{2} \cong 5 \mathrm{~s}$, to a stationary point of coordinates $(y=0, z=0)$. This result is confirmed by behaviors of system energy representations related to $V_{T}$ and $V_{I S}$, as shown in Fig. 4 .

Second case. Let us now consider a targeted magnitude of energy $V_{I S}^{*}=3$ (i.e. $V_{I S}^{*} \neq 0$ ). As shown

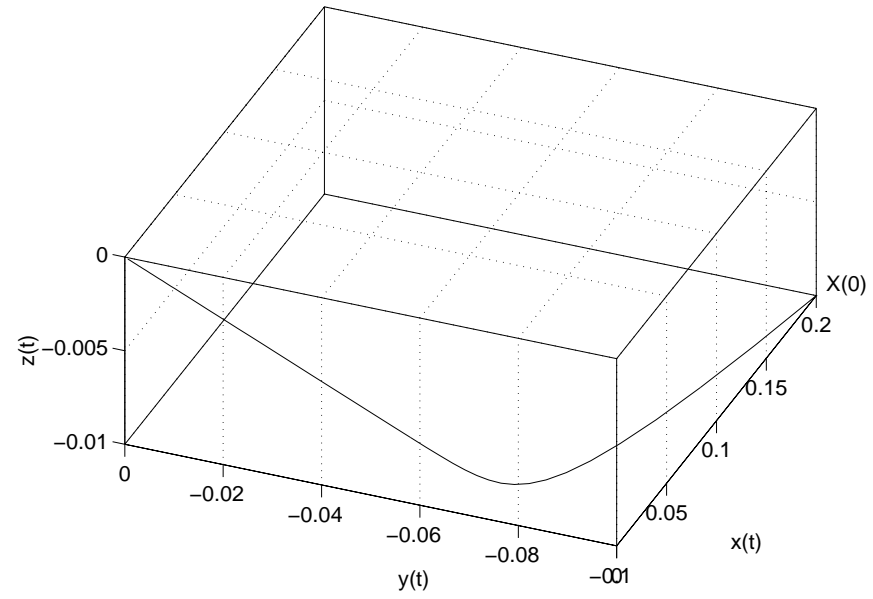

Fig. 2. Controlled system trajectory.

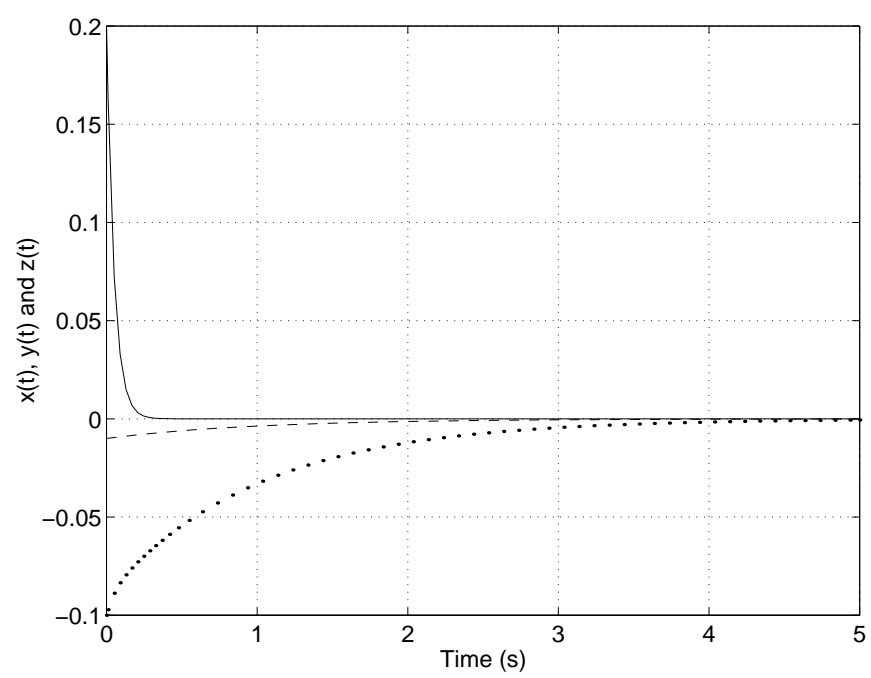

Fig. 3. Controlled state variables of Chua's circuit: $x(t)$ (solid), $y(t)$ (dotted), $z(t)$ (dashed).

in Fig. 5, the system trajectory also goes first to $(y, z)$ plane in finite time. However, for the present case, the system states reach a stationary point different from 0 on $(y, z)$ plane (see Fig. 6). Indeed, this point corresponds to the magnitude of $V_{I S}$ in a vicinity of $V_{I S}^{*}$, as shown in Fig. 7 .

Finally, to extend illustrations of ESMC approach capabilities, let us consider the results of Figs. 8-10, coming from appropriate modifications of control scheme $(5,6)$ according to Chua's circuit modeling and energy properties. These results show that such a scheme can lead to convergence of system trajectory to a stable limit cycle whom corresponding energy lies in the vicinity of the targeted magnitude $V_{I S}^{*}=3$ (see Fig. 10).

\footnotetext{
${ }^{5}$ Let us note that 0 is an unstable equilibrium point for the system (24).
} 
1408 L. Laval \& N. K. M'Sirdi
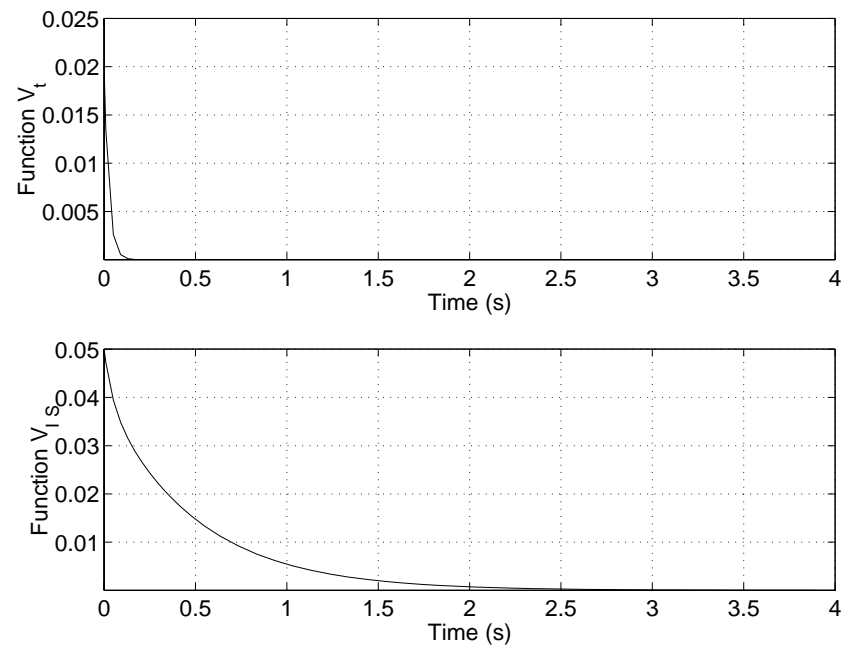

Fig. 4. Magnitude of $V_{T}$ and $V_{I S}$ versus time.

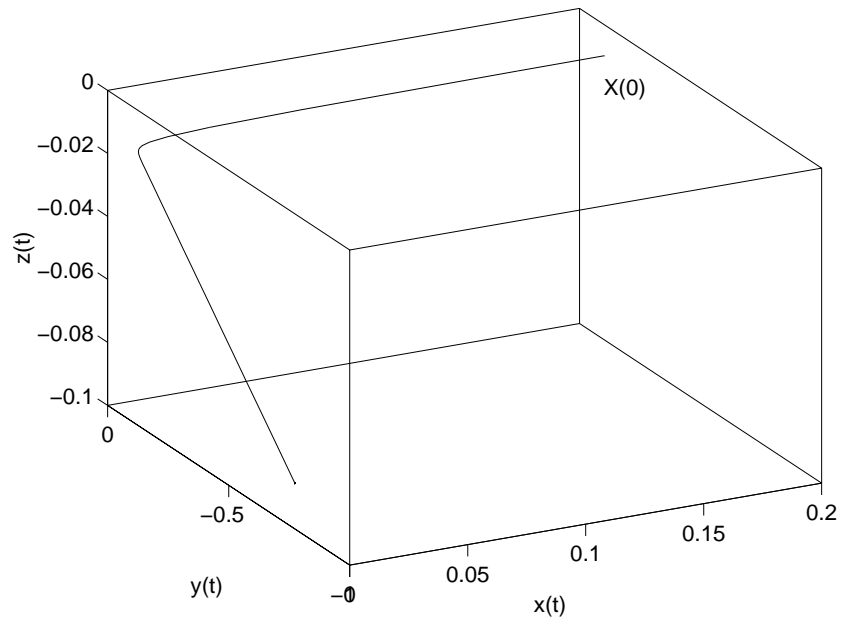

Fig. 5. Controlled system trajectory.

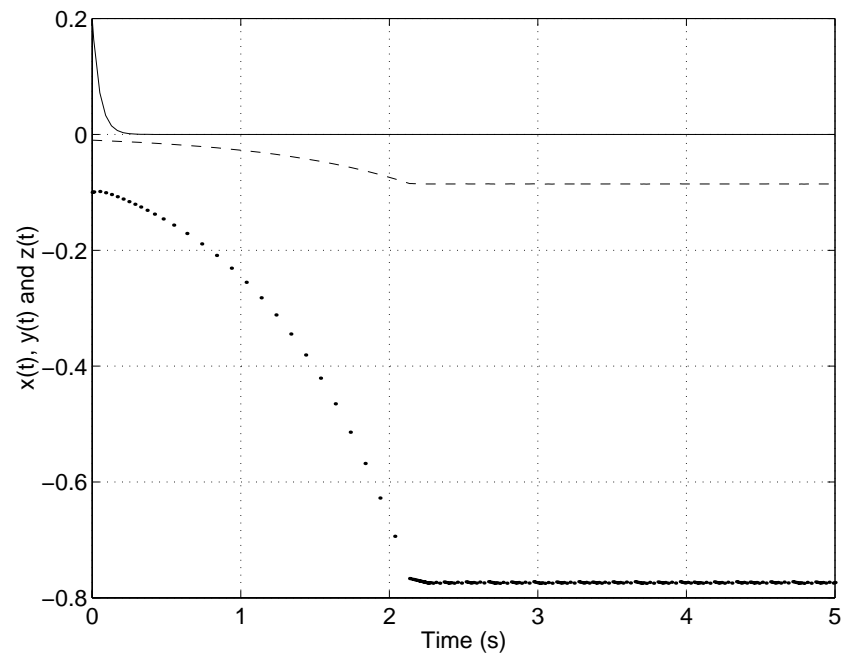

Fig. 6. Controlled state variables of Chua's circuit: $x(t)$ (solid), $y(t)$ (dotted), $z(t)$ (dashed).
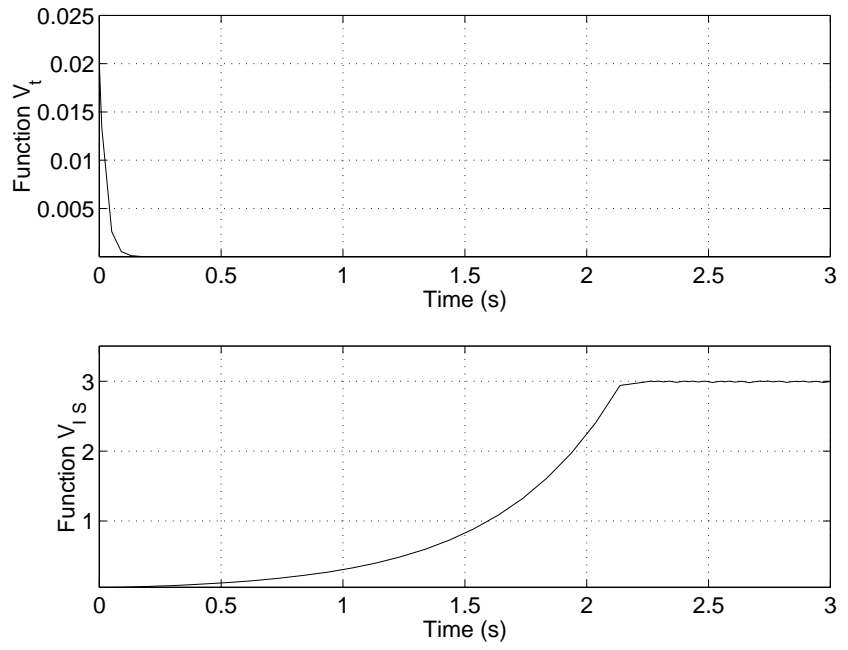

Fig. 7. Magnitude of $V_{T}$ and $V_{I S}$ versus time.

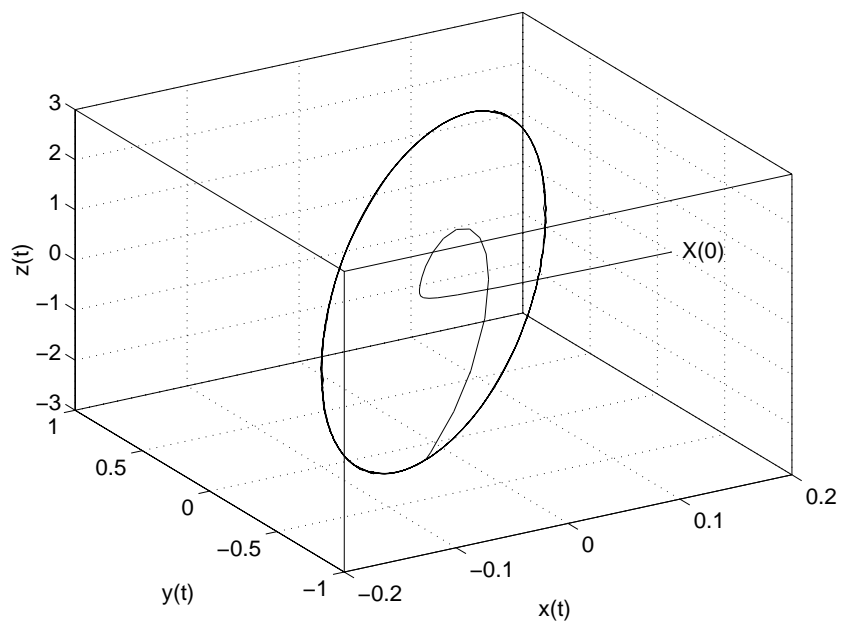

Fig. 8. Controlled system trajectory.

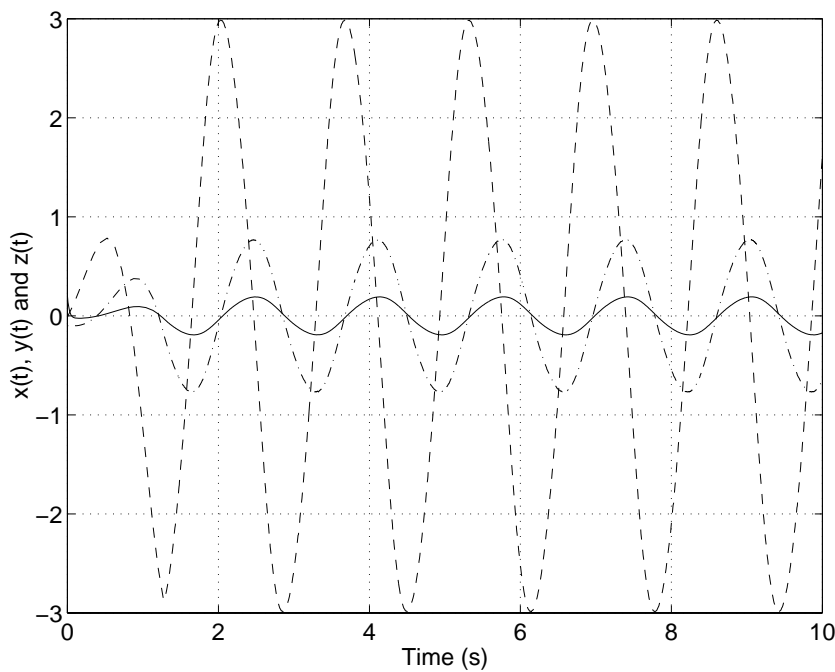

Fig. 9. Controlled state variables of Chua's circuit: $x(t)$ (solid), $y(t)$ (dashdot), $z(t)$ (dashed). 

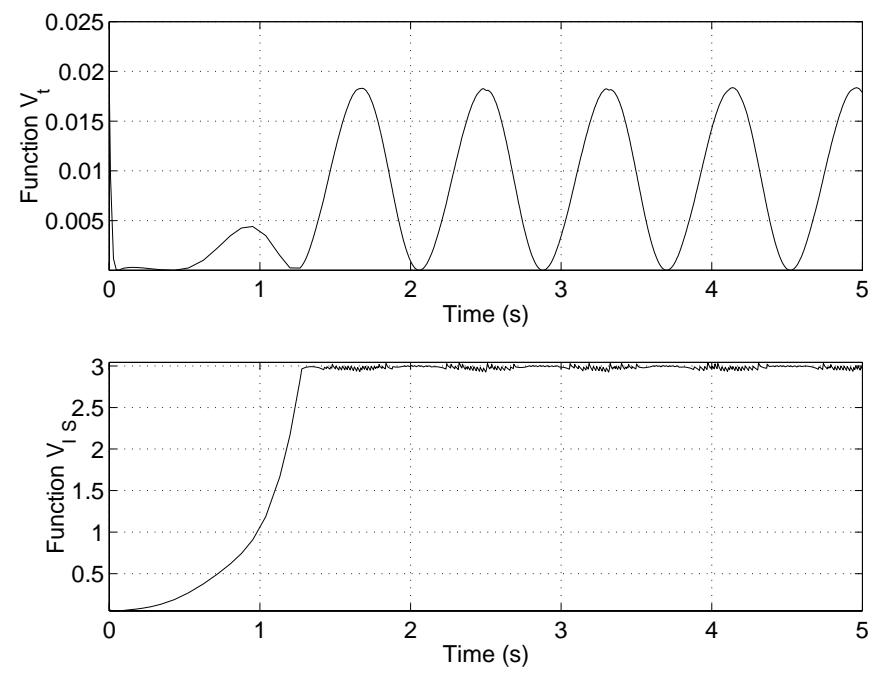

Fig. 10. Magnitude of $V_{T}$ and $V_{I S}$ versus time.

\section{Conclusion}

We have proposed a new control approach for a class of nonlinear systems fullfilling Assumptions A1-A3. This approach considers the system energy as basis for both control design and system stabilization. Application of this method to three-dimensional chaotic systems leads to extended capabilities compared to classical VSS controllers. Indeed, systems trajectories can be driven toward and maintained into global invariant sets without explicit definition of targeted orbits or stationary points. We think that this method can be extended to systems of higher dimension. This will be the objective of our future investigation.

\section{References}

Chen, G. \& Dong, X. [1998] From Chaos to Order: Perspectives, Methodologies, and Applications (World Scientific, Singapore).

Chen, G. [1999] Controlling Chaos and Bifurcations in Engineering Systems (CRC Press).

DeCarlo, R. A., Zak, S. H. \& Drakunov, S. V. [1996] "Variable structure, slidind-mode controller design," in The Control Handbook, Elec. Eng. Handbook Series (CRC Press).

Ditto, W. L., Spano, M. L. \& Lindner, J. F. [1995] "Techniques for the control of chaos," Physica D86(1\&2), 198-211.

Lenz, H. \& Berstecher, R. [1997] "Sliding-mode control of chaotic pendulum: Stabilization and targeting of an instable periodic orbit," in Control of Oscillations and Chaos Conf., Vol. 3, pp. 586-589.

Madan, R. [1993] Chua's Circuit: A Paradigm for Chaos, World Scientific Series on Nonlinear Science, Series B, Special Theme Issues and Proceedings, Vol. 1 (World Scientific, Singapore).

Slotine, J. J. E. \& Li, W. [1991] Applied Nonlinear Control (Prentice Hall, Englewood Cliffs, NJ).

Utkin, V. I. [1997] "Variable structure systems with sliding mode: A survey," IEEE Trans. Automat. Contr. AC-22(2), 212-222.

Yang, T. \& Chua, L. O. [1998] "Control of chaos using sampled-data feedback control," Int. J. Bifurcation and Chaos 8(12), 2433-2438.

Yau, H. T., Chen, C. K. \& Chen, C. L. [2000] "Sliding mode control of chaotic systems with uncertainties," Int. J. Bifurcation and Chaos 10(5), 1139-1147.

Yu, X. [1996] "Controlling Lorenz chaos," Int. J. Syst. Sci. 27(4), 355-359. 